\title{
Modelling change in multivariate depression symptoms adjusting for gender and baseline temperament and character traits: a Latent Transition approach
}

\author{
$\underline{\text { I.L. Hudson }}^{\text {a }}$, Lucy Leigh ${ }^{\text {a }}$ and Peter Joyce ${ }^{b}$ \\ ${ }^{a}$ School of Mathematical and Physical Sciences, The University of Newcastle, New South Wales, \\ ${ }^{b}$ Department of Psychological Medicine, Christchurch School of Medicine and Health Sciences, \\ University of Otago, New Zealand
}

Email: irene.hudson@newcastle.edu.au; irenelena.hudson@gmail.com

\begin{abstract}
Personality is linked to mental illness. Data on 9 symptoms of psychological distress items of the Symptom Checklist (SCLs) (Derogatis, 1983) and 7 temperament and character traits (TCIs) of Cloninger (2008) were analysed from patients measured pre and post treatment from the NZ Christchurch Psychotherapy of Depression Study (Joyce et al. 2002; Turner, Hudson et al., 2003). Latent transition analysis (LTA) was used to investigate: How do depressive symptom patterns change over time pre and post treatment; is this change different across gender and how is it impacted by baseline temperament and character traits (TCIs).

The LTA model found 4 latent classes based on the multivariate symptom response profiles of 9 SCLs. The 4 patient classes represent different levels of symptom risk whose interpretation varies across gender. Latent class 1 (C1), a high risk group was characterised by high probabilities of elevated scores on all 9 SCL items. Class 2 (C2) a low risk group was characterised by a low risk of high scores on all SCLs. In fact the likelihood of scoring above the median for depression, phobic anxiety and anxiety was zero for both sexes in C2. Class 4 males were characterised by very high depression, interpersonal sensitivity (IS), psychoticism (P) and OC, intermediate anxiety (A), phobic anxiety (PA) and paranoid ideation (PI) but low scores on somatisation (S). In contrast $\mathrm{C} 4$ females had a high risk of elevated anxiety and $\mathrm{P}$, intermediate $\mathrm{OC}$, depression and $\mathrm{S}$, but low scores on IS, anger hostility (AH), PI and PA. Class 3 females exhibited very high IS, high P, intermediate AH, $\mathrm{PI}$ and depression risk, and low scores of (phobic) anxiety. Class 3 males had very high $\mathrm{P}$ and intermediate $\mathrm{S}$ risk, and like C3 females low scores on IS, AH and PA. Anxiety differentiated females across C3 and C4. Apart from the high risk class, $\mathrm{C} 1$, only $\mathrm{C} 4$ females had increased anxiety risk. The latent classes seem to represent a scale of distress risk, suggesting a continuum of low to high risk, as opposed to qualitatively different groups.
\end{abstract}

LTA transition probabilities pre to post treatment showed the males had a higher chance of remaining in the high risk class than females. For both sexes at high risk pre-treatment, $0 \%$ transitioned from $\mathrm{C} 1$ to $\mathrm{C} 4$ posttreatment. For females at high risk pre-treatment, $37 \%$ moved to low risk, but only $20 \%$ males moved from high to low risk. Neither gender transitioned from $\mathrm{C} 4$ to $\mathrm{C} 3$. Three times more males in $\mathrm{C} 3$ remained in $\mathrm{C} 3$ (very high P, intermediate somatisation, low scores on IS, AH and PA) compared to females. The proportion of males remaining in C4 (high depression, IS, psychoticism and OC, and intermediate A, PA and PI) was over twice the $\mathrm{C} 4$ female rate (high anxiety and $\mathrm{P}$, intermediate $\mathrm{OC}$ and depression). No males starting in the low risk class transitioned to the high risk class, compared to $6 \%$ of females, whereas of females starting in the low risk group a quarter transitioned to $\mathrm{C} 4$. Three times more males starting in $\mathrm{C} 3$ transitioned to the high risk class than for $\mathrm{C} 3$ females, and more than twice as many males moved from $\mathrm{C} 3$ to $\mathrm{C} 4$. A quarter of females starting in $\mathrm{C} 4$ transitioned to the high risk class, but no $\mathrm{C} 4$ males moved to the high risk class $\mathrm{C} 1$ post-treatment.

Two character traits, self-directedness (SD) and self-transcendence (ST) were highly significant predictors of latent class status. In general higher ST was associated with higher distress and higher SD with lower distress. The impact of ST and SD varied across gender in class 4 and to a lesser extent in the low risk class. There was an enhanced benefit of increased SD for both sexes, especially for females in C4 characterised by a high risk of anxiety and P, intermediate OC, depression and S, but low scores on IS, AH, PI and PA, and for males in the low risk class. Increasing SD did not benefit the $\mathrm{C} 4$ males characterised by very high depression, IS, OC, and P, intermediate phobic/anxiety and PI, but low somatisation. Similar for both sexes, high ST was associated with higher odds of distress, except for males in C4. Low risk females were less likely to be in the low risk class ( $v s$ high) for a one unit increase in ST, and C2 males had a slightly less reduced odds of low risk vs 'high' risk status. This work extends Leigh et al. (2012) and shows that 4 discernible classes differed across gender, and transitions between classes pre to post treatment differed across gender (after baseline TCI adjustment). This study adds credence to the need for gender-specific scores of psychological distress risk to be developed.

Keywords: Latent transition analysis, psychological distress, temperament and character traits, gender 


\section{INTRODUCTION}

Personality is linked to mental illness (Joyce \& Mitchell, 2004). The relationship between symptoms of psychological distress (SCLs) and the temperament and character traits (TCIs) of Cloninger (2008) was recently investigated using latent class analysis (LCA) by Leigh et al., (2012). Multivariate SCL symptom profiles of 9 dimensions both pre- and post-treatment were regressed against 7 TCIs, with all predictor and symptom variables dichotomised by the median, Gender was included as testing for gender differences in predicting symptoms from TCIs has become popular (e.g. see the earlier univariate gender and time-specific generalised additive models (GAMs) of Turner (2004) which allowed for non-linearity between TCIs and SCLs). Leigh et al., (2012) identified three classes of patients both for pre- and post-treatment. The so-called 'high risk' and 'low risk' groups, were characterised by a small number of unique SCL response patterns. The most common pattern pre-treatment for the high risk group was high scores on all 9 SCLs, except somatisation and interpersonal sensitivity; and for post-treatment high scores on all SCLs, except obsessive compulsive and phobic anxiety. Leigh et al., (2012) also showed that in general higher self-transcendence and harm avoidance is associated with higher distress, and that the genders have a differential risk depending on levels of reward dependence and cooperativeness. In this current study LTA is used to investigate: how depressive symptoms change from pre and post treatment; how any change differs across gender and how is it impacted by all baseline TCIs. LTA classes thus correspond to discernible multivariate patterns of change from pre to post treatment. This work extends that of Leigh et al., (2012) from LCA to LTA allowing estimation of transition probabilities between classes across pre to post treatment, adjusted for baseline TCI traits and gender; the meaning of the classes can thus differ across gender.

\section{METHODS}

\subsection{Psychological distress symptom (SCL) and temperament and character trait (TCI) data}

The data analysed was based on combining two datasets from the Christchurch Outcome of Depression Study and Christchurch Psychotherapy of Depression Study (Joyce, Mulder et al. 2002). The first was a randomised controlled trial (RCT) of two antidepressants (fluoxetine or nortriptyline), the second a RCT of two psychotherapies (interpersonal psychotherapy and cognitive therapy). There was a total of 346 patients pretreatment, and 202 post-treatment. The study was based on 9 SCLs and 7 TCIs measured pre and post treatment for $\mathrm{N}=202$ patients, $66 \%$ females and 34\% males. The 9 symptoms from the Symptom Checklist (SCLs) (Derogatis, 1983) assessed pre- and post-treatment were: somatisation (S), obsessive compulsive (OC), interpersonal sensitivity (IS), depression (D), anxiety (A), anger hostility (AH), phobic anxiety (PA), paranoid ideation (PI) and psychoticism (P). The 7 TCIs assessed pre- and post- treatment were: the 4 temperament TCIs (novelty seeking (NS), harm avoidance (HA), reward dependence (RD) and persistence (P)) and 3 character traits (self-directedness (SD), cooperativeness (C) and self-transcendence (ST)).

\subsection{Analytic methods}

Multivariate SCL symptom profiles of 9 dimensions across pre- and post-treatment were analysed using LTA (http://methodology.psu.edu). Methods for estimation of transition probabilities between latent classes (statuses), pre and post treatment, adjusted for baseline TCIs and gender are shown below. SCL symptoms were dichotomised above and below the median (separately pre and post-treatment). Latent class models on the $9 \mathrm{SCLs}$ were run first, to determine the optimal number of latent classes using BIC, AIC criteria. The symptom item-response probabilities were not constrained to be equal across gender, allowing the meaning of the latent classes to differ across gender. TCI covariates were included as continuous predictors of latent status prevalence pre-treatment and for transitions. The baseline model was fitted first, gender was trialled as the grouping variable and tested for measurement invariance, before adding TCI covariates to the LTA.

Latent transition models extend LCA (Collins \& Lanza, 2010) to modelling both the latent class structures, and the transitions between these classes over time. Rather than model the entire vector of times simultaneously for an individual, transitions from one time to the next are estimated. Let there be $\mathrm{j}$ observed variables, $\mathrm{j}=$ $1, \ldots \mathrm{J}$, and the $\mathrm{J}$ observed variables are measured at time $\mathrm{t}=1, \ldots, \mathrm{T}$. Each observed variable $\mathrm{j}$ has $\mathrm{r}_{\mathrm{j}, \mathrm{t}}=1, \ldots, \mathrm{R}_{\mathrm{j}, \mathrm{t}}$ response categories. For simplicity, assume $R_{j, t}$ are constant over time, such that $R_{j, 1}=R_{j, 2}=\ldots R_{j, t}=R_{j}$, for all $\mathrm{j}$. W is the cross-table, formed by the $\mathrm{J}$ variables at $\mathrm{T}$ times,

$$
W=\prod_{t=1}^{T} \prod_{j=1}^{J} R_{j}
$$

Each cell of $\mathrm{W}$ corresponds to a complete response pattern, $\mathbf{y}=\left(\mathrm{r}_{11}, \ldots, \mathrm{r}_{\mathrm{j}, \mathrm{t}}\right)$. Y has W rows and (JxT) columns. Again a probability $\mathrm{P}(\mathbf{Y}=\mathbf{y})$ is associated with each pattern $\mathbf{y}$, where $\sum P(\boldsymbol{Y}=\boldsymbol{y})=1$. L is the overall latent 
categorical variable, with $\mathrm{S}$ latent statuses. $\mathrm{L}_{1}$ is the categorical latent variable at time 1 , where $\mathrm{s}_{1}=1, \ldots, \mathrm{S}$. $\mathrm{L} 2$ is the categorical latent variable at time 2 , where $\mathrm{s}_{2}=1, \ldots, \mathrm{S}$, and so on up to $\mathrm{L}_{\mathrm{T}}$ at time $\mathrm{T}$, where $\mathrm{s}_{\mathrm{T}}=1, \ldots, \mathrm{S}$. For simplicity, let $\mathrm{s}_{1}=\mathrm{s}_{2}=\ldots=\mathrm{S}_{\mathrm{T}}=\mathrm{S}$ (i.e. same number of classes at each time). There are three sets of parameters in the latent transition model; the latent statuses $(\delta)$, the transition probabilities $(\tau)$ and the item response probabilities $(\rho)$, where, $\delta_{\text {st }}$ is the prevalence of latent status s at time $t$ (i.e. the probability of belonging to latent status s at time t), and an individual can only belong to one status, $\sum_{s_{t}=1}^{S} \delta_{s_{t}}=1, \rho_{j r_{j} t \mid s_{t}}$ is the probability of response $r_{j t}$ on observed variable $\mathrm{j}$, conditional on belonging to latent status $\mathrm{s}$ at $\mathrm{t}$. An individual can only have one response per item, $\sum_{r_{j, t}=1}^{R_{j}} \rho_{j, r_{j}, t \mid s_{t}}=1$ (assuming item response probabilities are equal across time as previously stated) for $\tau_{s_{t+1} \mid s_{t}}$, the probability of transitioning to latent status $\mathrm{s}$ at $\mathrm{t}+1$, given membership in latent status $\mathrm{s}$ at $\mathrm{t}$, an individual can only transition to one status, $\sum_{s_{t+1}=1}^{S} \tau_{s_{t+1} \mid s_{t}}=1$. Let $\mathrm{I}\left(\mathrm{y}_{\mathrm{jt}}=\mathrm{r}_{\mathrm{jt}}\right)$ be an indicator variable, equal to 1 when $y_{j}=r_{j}$ at time $t$, and 0 otherwise. The probability of observing a particular vector of responses can then be expressed as a function of the probability of membership in each of the latent statuses at time $1\left(\delta_{\mathrm{s} 1}\right.$ 's), the probability of transitioning to a latent status at a particular time conditional on the latent status membership at the time immediately previous ( $\tau$ 's), and the probability of observing each response at each time conditional on the latent status membership ( $\rho$ 's) such that

$$
P(\mathbf{Y}=\mathbf{y})=\sum_{s_{1}=1}^{S} \ldots \sum_{S_{T}=1}^{S} \delta_{s_{1}} \tau_{s_{2} \mid s_{1}} \ldots \tau_{s_{T} \mid s_{T-1}} \prod_{t=1}^{T} \prod_{j=1}^{J} \prod_{r_{j}, t=1}^{R_{j}} \rho_{j r_{j} t \mid s_{t} .}^{I\left(y_{j t}=r_{j t}\right)}
$$

Degrees of freedom (df) in the LTA models are as follows, $\mathrm{df}=\mathrm{W}-\mathrm{P}_{\delta}-\mathrm{P}_{\rho}-\mathrm{P}_{\tau}-1$ for $\mathrm{P}_{\delta}=$ number of latent status prevalences estimated, $\mathrm{P}_{\rho}=$ number of item response probabilities estimated, $\mathrm{P}_{\tau}=$ number of transitions estimated. In regard to the number of latent statuses $\mathrm{P}_{\delta}$, only the time 1 values are estimated independently (the rest calculated from the transitions: e.g. $\delta_{s t}=\sum_{s_{t-1}=1}^{S} \delta_{s_{t-1}} \tau_{s_{t} \mid s_{t-1}} ; \mathrm{P}_{\delta}=\mathrm{S}-1$ (if no restrictions are placed on the classes), $\mathrm{P}_{\rho}=S T \sum_{j=1}^{J} R_{j-1}$ and $\mathrm{P}_{\tau}=(\mathrm{T}-1) \mathrm{S}(\mathrm{S}-1)$, The df in LTA models needs to be large, since $\mathrm{W}$ is large, but there are often identification problems due to sparseness. Constraining the statuses to be equal over time helps to solve the identification issues. Use of the AIC and BIC is recommended for comparing a variety of numbers of classes (Collins and Lanza, 2011). In the multi-group LTA (here, testing gender) we define $j, t, r_{j}$, $\mathrm{L}$ etc. as before, and let $\mathrm{V}$ be the grouping variable with $\mathrm{q}=1, \ldots, \mathrm{Q}$ groups. For simplicity, constrain the number of statuses and response categories to be constant over time, such that

$$
W=Q \cdot \prod_{t=1}^{T} \prod_{j=1}^{J} R_{j}
$$

The vector $\mathbf{y}=\left(\mathrm{q}, \mathrm{r}_{1,1}, \ldots, \mathrm{r}_{\mathrm{J}, \mathrm{T}}\right)$ in $(2.3)$ corresponds to the response pattern in cell w, with probability $\mathrm{P}(\mathbf{Y}=\mathbf{y})$ and $\sum P(\boldsymbol{Y}=\boldsymbol{y} \mid V=q)=1$, for

$$
P(\mathbf{Y}=\mathbf{y} \mid \mathrm{V}=\mathrm{q})=\sum_{s_{1}=1}^{S} \ldots \sum_{S_{T}=1}^{S} \delta_{s_{1} \mid q} \tau_{s_{2} \mid s_{1}, q} . \tau_{s_{T} \mid s_{T-1}, q} \prod_{t=1}^{T} \prod_{j=1}^{J} \prod_{r_{j}, t=1}^{R_{j}} \rho_{j r_{j} t \mid s_{t}, q .}^{I\left(y_{j t}=r_{j t}\right)} .
$$

TCI baseline (here, as pre-treatment) covariates can be added to predict membership in different latent statuses and/or transitions between the latent statuses. Note, ' $j$ ' are the observed indicator, at ' $t$ ' times, with $r_{j}$ response categories (assumed equal over time for simplicity), Let $\mathrm{L}_{t}$ be the categorical latent variable at time $\mathrm{t}$ with $\mathrm{S}$ latent statuses (number held equal across time for simplicity). Let $\mathrm{X}$ be the (TCI) covariate, in this case predicting time 1, pre-treatment latent statuses and the transitions,

$$
P(\mathbf{Y}=\mathbf{y} \mid \mathbf{X}=\mathbf{x})=\sum_{s_{1}=1}^{S} \ldots \sum_{S_{T}=1}^{S} \delta_{s_{1}}(x) \tau_{s_{2} \mid s_{1}}(x) \ldots \tau_{s_{T} \mid s_{T-1}}(x) \prod_{t=1}^{T} \prod_{j=1}^{J} \prod_{r_{j}, t=1}^{R_{j}} \rho_{j r_{j} t \mid s_{t}}^{I\left(y_{j t}=r_{j t}\right)},
$$

where $\delta_{S_{1}}(x)=P\left(L_{1}=S_{1} \mid X=x\right)$ and $\delta_{s_{1}}(x)=P\left(L_{1}=S_{1} \mid X=x\right)$ are standard baseline-category multinomial logistic regressions. Prediction for latent status membership at any time is as follows,

$$
\delta_{s_{t}}(x)=\frac{e^{\beta_{0_{s}}+\beta_{1_{s}} x}}{1+\sum_{s_{1^{\prime}=1}}^{S-1} e^{\beta_{0_{s_{t}}}+\beta_{1_{s}} t^{x}}} .
$$

As each row of the transition probability matrix sums to 1 , see equation (2.5), when a covariate predicts transitions, a separate regression is used for each row, so each row has its own reference category. For example, for the first row of the transition matrix with a single covariate $X, \boldsymbol{\tau}_{\boldsymbol{s}_{t} \mid \mathbf{1}_{t-1}}(\boldsymbol{x})$ the transition probability for those who were in latent status 1 at time $\mathrm{t}-1$, is

$$
\tau_{s_{t} \mid s_{t-1}}(x)=\frac{e^{\beta_{0} s_{t} \mid s_{t-1}}+e^{\beta_{s_{t} \mid s_{t-1}} x}}{1+\sum_{s_{1^{\prime}=1}}^{S-1} e^{\beta_{s_{t} \mid s_{t-1}}+e^{\beta_{1} \mid s_{t-1} x}} .}
$$




\section{RESULTS}

Table 1. Pre and post treatment medians of SCLs

\begin{tabular}{|l|c|c|c|c|}
\hline \multicolumn{1}{|c|}{ Distress Symptoms (SCLs) } & \multicolumn{2}{|c|}{ Pre treatment } & \multicolumn{2}{c|}{ Post treatment } \\
\hline & Male & Female & Male & Female \\
\hline Somatisation (S (SCL)) & 0.9 & 0.8 & 0.3 & 0.3 \\
\hline Obsessive compulsive (OC) & 1.9 & 1.6 & 0.8 & 0.4 \\
\hline Interpersonal sensitivity (IS) & 1.6 & 1.5 & 0.7 & 0.2 \\
\hline Depression (D) & 2.3 & 2.3 & 0.9 & 0.5 \\
\hline Anxiety (A) & 1.4 & 1.3 & 0.5 & 0.3 \\
\hline Anger hostility (AH) & 1.0 & 1.0 & 0.2 & 0.2 \\
\hline Phobic anxiety (PA) & 0.5 & 0.4 & 0.0 & 0.0 \\
\hline Paranoid ideation (PI) & 1.7 & 1.0 & 0.5 & 0.2 \\
\hline Psychoticism (P) & 1.0 & 0.8 & 0.3 & 0.1 \\
\hline
\end{tabular}

High SCL symptom scores indicate increased distress. Table 1 shows that post treatment SCL scores have decreased for both genders, with females, both pre and post treatment scoring lower than males on most SCLs. Although TCI scores are not reported, pretreatment TCIs were treated as continuous predictors in the LTA, initially scaled from 0 to 1 , then rescaled 0 to 10 and centred at 5 to assist interpretation of the predicted odds ratios of high SCLs (>median) across classes.

The LTA model using gender as a grouping variable, both with and without adjustment of baseline TCIs, found 4 latent classes based on the multivariate profiles of 9 SCLs (Table 3 LHS and RHS). We report in detail the TCI covariate adjusted LTA model for which self-directedness (SD) and self-transcendence (ST) were selected as significant predictors via backwards stepwise procedures. Gender specific item response probabilities (IRPs) for this model give the probability of scoring above the median on each SCL, conditional on class membership (Table 2). For both genders latent status class 1 (C1, a high risk group) is characterised by high probabilities of elevated scores on all SCL items. C1 comprised 28\% males and females pre- treatment, 34\% males vs 23\% females post-treatment. Class $2(\mathrm{C} 2$, a low risk group), with $6 \%$ males and $36 \%$ females pre-treatment, $28 \%$ males $v s 49 \%$ females post treatment, was characterised by a low likelihood of scoring high on all SCL items. Indeed the likelihood of high scores of depression, phobic anxiety and anxiety was zero for all patients in $\mathrm{C} 2$. Class 3 (C3) males, 49\% pre- vs 2\% post-treatment, scored low on interpersonal sensitivity (IS) (0.13), anger hostility (AH) (0.33), phobic anxiety (PA) (0.29) and anxiety (0.43), but had a high chance of elevated psychoticism (0.91) and an intermediate risk of higher somatisation (0.51). Similarly females in C3 (10\% preand post-treatment) exhibited low scores of phobic anxiety (PA) (0.26), with a zero likelihood of elevated anxiety (0.00), but exhibited elevated psychoticism (0.67), but lower than the C3 males. However, unlike the C3 males, C3 females had a high risk of increased interpersonal sensitivity (IS) (0.94), and an intermediate risk of both anger hostility (0.60) and paranoid ideation (PI) (0.59), but low risk of increased somatisation (S) (0.25). Class 4 males, $17 \%$ pre- vs $27 \%$ post-treatment, had a very high risk of elevated symptoms on all of depression (0.93), IS (0.92), psychoticism (0.90) and OC (0.85), and an intermediate risk of increased phobic/anxiety (0.55). In contrast C4 females, $27 \%$ pre- $v s 18 \%$ post treatment, were at risk of higher anxiety (0.70) and psychoticism (0.65), but not an increased risk of depression nor OC symptoms like the C4 males. For C4, only females had elevated anxiety. Pre-treatment C4 comprised $17 \%$ males vs $27 \%$ females, and posttreatment, $27 \%$ males vs $18 \%$ females. LTA IRPs by gender for the LTA model without adjustment for baseline TCIs are given in Figure 1 for comparison to the covariate adjusted model (compare Table 4 with Table 3).

Gender-specific transition probabilities between latent classes pre to post treatment are given in Table 5. The percentage of males in the high distress class $(\mathrm{C} 1)$ pre-treatment, who remain at high risk, was greater for males $(68 \%)$ than females $(50 \%)$. All males in the low risk class $(\mathrm{C} 2)$ pre-treatment remained at low risk, $100 \%$ compared to $66 \%$ of $\mathrm{C} 2$ females remaining at low risk post treatment. Three times more males starting in $\mathrm{C} 3$ (characterised by high psychoticism, intermediate somatisation) remained in $\mathrm{C} 3,53 \%$ compared to $18 \%$ females (high psychoticism and IS, intermediate AH, PI and depression). The proportion of males remaining in C4 (high depression, IS, psychoticism and OC, intermediate anxiety, phobic anxiety, PI) was high (74\%), with fewer females, 30\%, staying in $\mathrm{C} 4$ (high anxiety and psychoticism, intermediate OC and depression). For females at high risk (C1) pre-treatment, 37\% transitioned to low risk, 13\% to C3 (high IS and psychoticism, intermediate $\mathrm{AH}, \mathrm{PI}$ and depression) and $0 \%$ to $\mathrm{C} 4$ (high anxiety and psychoticism, intermediate OC class). Whilst for males starting at high risk $(\mathrm{C} 1)$, a lower proportion, $20 \%$, transitioned to the low risk class, $12 \%$ to C3 (with post treatment reduced OC, IS, anger hostility, phobic anxiety, anxiety, depression and PI, but remaining high risk on psychoticism and intermediate on somatisation), and like the $\mathrm{C} 1$ females, no males transitioned from high risk to $\mathrm{C} 4$ (characterised by high depression, IS, psychoticism and OC, intermediate anxiety, PA and PI) post-treatment. No males starting in the low risk group transitioned to the high risk class post-treatment, compared to $6 \%$ of females. For females starting in the low risk group (C2) $3 \%$ and $25 \%$ transitioned to $\mathrm{C} 3$ and $\mathrm{C} 4$, respectively, compared to no males (i.e. the low risk males pre-treatment remained 
Hudson et al., Modelling change in multivariate depression symptoms adjusting for gender: a LTA approach

Table 2. Males SCL Response category 2 (> median) with baseline covariates SD and ST

\begin{tabular}{lccccccccc}
\hline Class: & High & Low & C3 & C4 & Class: & High & Low & C3 & C4 \\
\hline SSCL & $\mathbf{0 . 9 6 3 7}$ & 0.1495 & 0.5123 & 0.2587 & SSCL & $\mathbf{0 . 8 2 3 6}$ & 0.1694 & 0.2484 & 0.5045 \\
OCSCL & $\mathbf{0 . 9 5 3 1}$ & 0.0366 & 0.3604 & $\mathbf{0 . 8 4 5 8}$ & OCSCL & $\mathbf{0 . 9 0 9 1}$ & 0.0781 & 0.3249 & 0.5627 \\
ISSCL & $\mathbf{1 . 0 0 0 0}$ & 0.0878 & 0.1336 & $\mathbf{0 . 9 2 0 2}$ & ISSCL & $\mathbf{0 . 9 5 7 1}$ & 0.0549 & $\mathbf{0 . 9 4 3 2}$ & 0.2232 \\
DSCL & $\mathbf{0 . 8 9 1 8}$ & 0.0000 & 0.2845 & $\mathbf{0 . 9 3 0 4}$ & DSCL & $\mathbf{0 . 9 8 3 6}$ & 0.0000 & 0.5410 & 0.5287 \\
ASCL & $\mathbf{1 . 0 0 0 0}$ & 0.1137 & 0.4322 & 0.5514 & ASCL & $\mathbf{0 . 8 9 0 6}$ & 0.0000 & 0.0000 & $\mathbf{0 . 6 9 5 4}$ \\
AHSCL & $\mathbf{0 . 8 3 9 9}$ & 0.0434 & 0.3260 & 0.3658 & AHSCL & 0.6564 & 0.1726 & $\mathbf{0 . 5 9 6 3}$ & 0.3838 \\
PASCL & $\mathbf{0 . 8 4 4 1}$ & 0.0000 & 0.2863 & 0.5510 & PASCL & $\mathbf{0 . 7 8 8 8}$ & 0.0768 & 0.2616 & 0.4481 \\
PISCL & $\mathbf{0 . 9 6 7 8}$ & 0.1128 & 0.4729 & 0.5068 & PISCL & $\mathbf{0 . 8 1 2 6}$ & 0.1260 & 0.5869 & 0.3474 \\
PSCL & $\mathbf{0 . 9 7 6 1}$ & 0.0000 & $\mathbf{0 . 9 0 8 8}$ & $\mathbf{0 . 8 9 4 9}$ & PSCL & $\mathbf{1 . 0 0 0 0}$ & $\mathbf{0 . 1 7 5 5}$ & $\mathbf{0 . 6 7 3 2}$ & $\mathbf{0 . 6 4 9 7}$
\end{tabular}

Table 3.

Delta estimates (status membership probabilities)

with baseline covariates SD and ST

Group 1 (males):

\begin{tabular}{llllcllllll}
\hline Status: & High & Low & C3 & C4 & Status: & High & Low & C3 & C4 \\
\hline Pre- & 0.2784 & 0.0595 & 0.4908 & 0.1713 & Pre- & 0.2817 & 0.1811 & 0.4020 & 0.1351 \\
Post- & 0.3439 & 0.2719 & 0.1192 & 0.2649 & Post- & 0.3514 & 0.2337 & 0.1628 & 0.2521 \\
\hline Group & $\mathbf{2}$ (females): & & & Group & $\mathbf{2}$ (females): & & \\
\hline Status & High & Low & C 3 & C4 & Status & High & Low & C3 & C4 \\
Pre- & 0.2762 & 0.3573 & 0.1013 & 0.2651 & Pre- & 0.2390 & 0.4085 & 0.1051 & 0.2474 \\
Post- & 0.2341 & 0.4891 & 0.0983 & 0.1785 & Post- & 0.2405 & 0.4686 & 0.1357 & 0.1552
\end{tabular}

Table 4.

Males SCL Response category 2 (> median)

no baseline covariates SD and $S T$

\begin{tabular}{lcccc}
\hline Class: & High & Low & C 3 & C4 \\
\hline SSCL & $\mathbf{0 . 9 7 2 5}$ & 0.0000 & $\mathbf{0 . 6 8 3 1}$ & 0.1886 \\
OCSCL & $\mathbf{0 . 9 5 6 4}$ & 0.0385 & 0.4370 & $\mathbf{0 . 8 4 1 1}$ \\
ISSCL & $\mathbf{1 . 0 0 0 0}$ & 0.0705 & 0.1928 & $\mathbf{0 . 9 3 7 7}$ \\
DSCL & $\mathbf{0 . 9 0 6 9}$ & 0.0000 & 0.3368 & $\mathbf{0 . 9 5 5 4}$ \\
ASCL & $\mathbf{1 . 0 0 0 0}$ & 0.1434 & 0.4623 & $\mathbf{0 . 5 4 3 5}$ \\
AHSCL & $\mathbf{0 . 8 3 7 0}$ & 0.1302 & 0.2844 & 0.3898 \\
PASCL & $\mathbf{0 . 8 4 3 5}$ & 0.1301 & 0.2530 & $\mathbf{0 . 5 4 0 8}$ \\
PISCL & $\mathbf{0 . 9 3 3 0}$ & 0.0918 & $\mathbf{0 . 5 3 8 0}$ & $\mathbf{0 . 5 5 9 4}$ \\
PSCL & $\mathbf{0 . 9 2 9 6}$ & 0.0653 & $\mathbf{0 . 5 1 5 5}$ & $\mathbf{0 . 8 1 8 8}$
\end{tabular}

Females SCL Response category 2 ( $>$ median) no baseline covariates $S D$ and $S T$

\begin{tabular}{lcrrr}
\hline Class: & High & Low & C3 & C4 \\
\hline SSCL & $\mathbf{0 . 8 4 6 3}$ & 0.1724 & 0.3161 & 0.5064 \\
OCSCL & $\mathbf{0 . 8 9 9 7}$ & 0.0890 & 0.3397 & 0.6288 \\
ISSCL & $\mathbf{0 . 9 5 3 8}$ & 0.0261 & $\mathbf{0 . 8 7 9 8}$ & 0.3233 \\
DSCL & $\mathbf{0 . 9 9 8 0}$ & 0.0118 & 0.4443 & 0.6172 \\
ASCL & $\mathbf{0 . 8 7 7 3}$ & 0.0000 & 0.0000 & $\mathbf{0 . 8 5 0 0}$ \\
AHSCL & $\mathbf{0 . 7 1 4 5}$ & 0.1748 & 0.5247 & 0.3678 \\
PASCL & $\mathbf{0 . 8 2 3 6}$ & 0.0904 & 0.2033 & $\mathbf{0 . 4 8 5 2}$ \\
PISCL & $\mathbf{0 . 8 3 1 5}$ & 0.1456 & $\mathbf{0 . 5 6 1 1}$ & 0.3255 \\
PSCL & $\mathbf{0 . 9 7 1 1}$ & 0.3161 & $\mathbf{0 . 4 0 0 9}$ & $\mathbf{0 . 3 8 6 6}$
\end{tabular}

Table 5. Transition probabilities with covariates

Males Pre- status (rows); Post- status (columns)

\begin{tabular}{lcccccccccc} 
Males & \multicolumn{1}{c}{ Pre- status (rows); Post- status (columns) } & \multicolumn{3}{c}{ Females } & Pre- status (rows); Post- status (columns) \\
\hline Class & High & Low & C3 & C4 & Class & High & Low & C3 & C4 \\
\hline High & $\mathbf{0 . 6 7 6 1}$ & $\mathbf{0 . 2 0 4 2}$ & 0.1196 & 0.0000 & High & $\mathbf{0 . 5 0 2 3}$ & $\mathbf{0 . 3 6 9 7}$ & 0.1279 & 0.0000 \\
\hline Low & 0.0000 & $\mathbf{1 . 0 0 0 0}$ & 0.0000 & 0.0000 & Low & 0.0575 & $\mathbf{0 . 6 6 4 0}$ & 0.0273 & $\mathbf{0 . 2 5 1 1}$ \\
C3 & $\mathbf{0 . 3 1 7 2}$ & 0.2261 & $\mathbf{0 . 1 7 5 1}$ & 0.2816 & C3 & 0.1150 & $\mathbf{0 . 2 4 9 4}$ & $\mathbf{0 . 5 2 5 4}$ & 0.1101 \\
C4 & 0.0000 & $\mathbf{0 . 2 6 0 5}$ & 0.0000 & $\mathbf{0 . 7 3 9 5}$ & C4 & 0.2380 & $\mathbf{0 . 4 6 9 4}$ & 0.0000 & $\mathbf{0 . 2 9 2 6}$
\end{tabular}

Table 6. Transition probabilities no covariates

Males Pre - status (rows): Post- status (columns) Females Pre - status (rows): Post- status (columns)

\begin{tabular}{|c|c|c|c|c|c|c|c|c|c|}
\hline Class & High & Low & C 3 & $\mathrm{C4}$ & Class & High & Low & $\mathbf{C 3}$ & C4 \\
\hline High & 0.6271 & 0.1006 & 0.2723 & 0.0000 & High & 0.4738 & 0.3410 & 0.1852 & 0.0000 \\
\hline Low & 0.0000 & 0.5572 & 0.0000 & 0.4428 & Low & 0.4738 & 0.3410 & 0.1852 & 0.0000 \\
\hline $\mathrm{C} 3$ & 0.4345 & 0.2087 & 0.2142 & 0.1425 & $\mathrm{C} 3$ & 0.1301 & 0.1637 & 0.5818 & 0.1244 \\
\hline $\mathrm{C} 4$ & 0.0000 & 0.1515 & 0.0000 & 0.8485 & $\mathrm{C} 4$ & 0.3435 & 0.5249 & 0.0000 & 0.1315 \\
\hline Table 7. & \multicolumn{4}{|c|}{ Betas for the covariates SD \& ST Males } & Females & & & & \\
\hline Status & High & Low & C3 & C4 & Status & High & Low & C3 & $\mathrm{C4}$ \\
\hline Intercept & Ref & -11.1719 & 1.1097 & 1.4622 & Intercept & Ref & -3.7720 & 0.6145 & -3.4322 \\
\hline $\mathrm{SD}$ & Ref & 15.1640 & 2.0666 & -5.3793 & $\mathrm{SD}$ & Ref & 12.1400 & 0.1931 & 8.3468 \\
\hline ST & Ref & -1.7569 & -4.7217 & 0.8510 & ST & Ref & -7.9616 & -5.6211 & -2.1117 \\
\hline
\end{tabular}

OR estimates of SD \& ST

Males

Females

\begin{tabular}{llllllllll} 
Status: & High & Low & C3 & C4 & Status: & High & Low & C 3 & C4 \\
\hline Intercept & Ref & 0.0000 & 3.0335 & 4.3157 & Intercept & Ref & 0.0230 & 1.8488 & 0.0323 \\
SD & Ref & 4.5558 & 1.2296 & 0.5840 & SD & Ref & 3.3669 & 1.0195 & 2.3041 \\
ST & Ref & 0.8389 & 0.6236 & 1.0888 & ST & Ref & 0.4511 & 0.5700 & 0.8096
\end{tabular}


at low risk). Of the males starting in $\mathrm{C} 3,32 \%$ transitioned to the high risk class (C1), almost three times more than for $\mathrm{C} 3$ females (12\%), and with more than twice as many males transitioning from $\mathrm{C} 3$ to $\mathrm{C} 4$ (28\% vs $11 \%$ females) post treatment. Note that neither gender transitioned from C4 to C3 post-treatment. Whilst $24 \%$ of females starting in $\mathrm{C} 4$ transitioned to the high risk class, no males transitioned from $\mathrm{C} 4$ to the high risk group. Also for the pre-treatment $\mathrm{C} 4$ membership, $47 \%$ females transitioned from $\mathrm{C} 4$ to the low risk class, almost twice that of males moving from $\mathrm{C} 4$ to the low risk (C2) post treatment, 26\%. Table 6 gives the transition probabilities for an LTA model with no baseline TCI covariate adjustment. The LTA without adjustment for baseline TCIs also found four classes, but their interpretation differs from the TCI-adjusted classes. The main difference with the unadjusted model (Table 6) is that of the males starting in the low risk $\mathrm{C} 2,44 \%$ transitioned to $\mathrm{C} 4$ compared to no females, and $47 \%$ of low risk $\mathrm{C} 2$ females moved to the high risk group, compared to no males. Whilst for the adjusted model (Table 5) $0 \%$ males moved from low risk C2 to C4 vs 25\% females, and $6 \%$ of low risk females transitioned to the high risk class post treatment, compared to $0 \%$ of males.
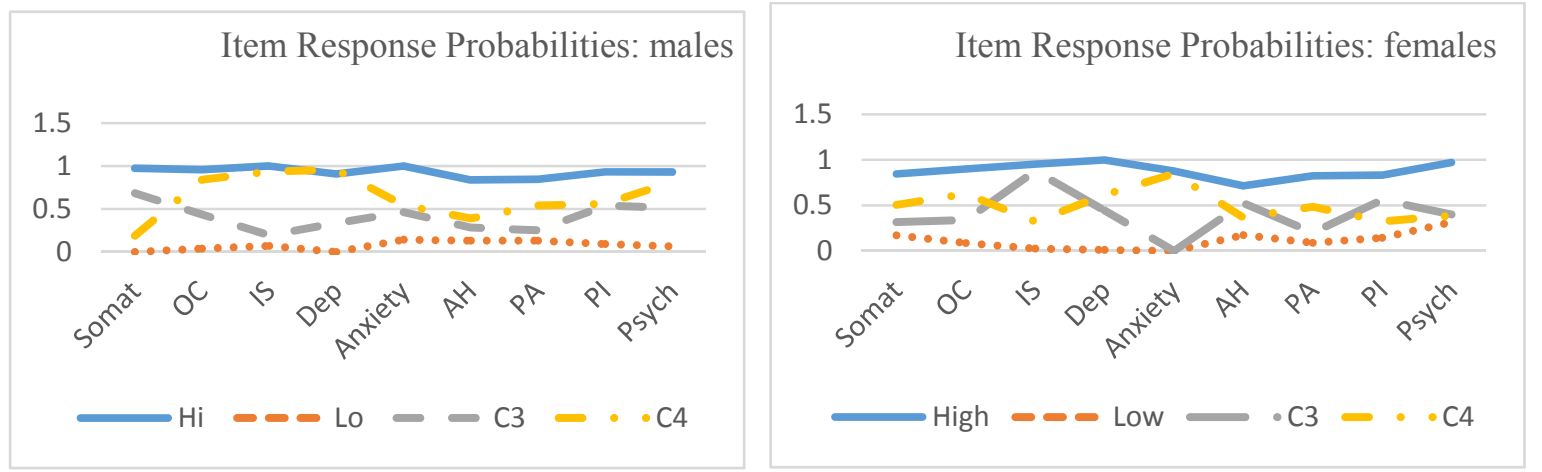

Figure 1. Item response probabilities by class and gender: LTA Model with no baseline TCI covariates.

Two pre-treatment TCIs were highly significant predictors of latent class status, self-directedness $(\mathrm{SD})(\mathrm{p}<0$. $00001)$ and self-transcendence $(\mathrm{ST})(\mathrm{p}<0.00002)$ (Table 7). Gender-specific beta estimates and odds ratios (ORs) of class membership $v s$ the high risk class (ref) are given in Table 7, respectively. For both sexes, increasing SD was associated with reduced symptoms (greater odds of being in a class of lower than 'high risk'), except for class 4 males. The odds of being in class 3 were roughly comparable to the high risk class for both sexes, but class 4 males had reduced odds $(\mathrm{OR}=0.58)$ of being in class 4 compared to high risk class; females had over twice the odds (2.3) of being in class 4 compared to the high risk class, for a one unit increase in selfdirectedness (Table 7). Males had over four times the odds (4.56) of being in the low risk class $v s$ the high risk, for a one unit increase in self-directedness, compared to females, with 3.37 odds for a one unit increase in SD. There was an enhanced benefit of increased SD for both genders, especially for females in C4 (high anxiety and psychoticism, intermediate $\mathrm{OC}$ and depression) and to a less extent males in the low risk class. Increasing SD does not seem to benefit the C4 males_(high depression, IS, psychoticism and OC, intermediate A, PA and PI). In both sexes, high levels of ST were associated with higher odds of distress, except for males in $\mathrm{C} 4(\mathrm{OR}=1.09)$ with such males having equal risk as the 'high risk' class for a one unit increase in ST. Low risk C2 females were less likely to be in the low class ( $v s$ high) for a one unit increase in ST, reduced odds $(\mathrm{OR}=0.45)$ compared to $\mathrm{C} 2$ males with $(\mathrm{OR}=0.84)$ of being in the low risk $v s$ 'high risk' for a one unit increase in ST (Table 7).

\section{DISCUSSION AND CONCLUSIONS}

The LTA model established 4 latent classes based on the 9 parameter multivariate symptom response (SCL) profiles. After adjustment for baseline TCIs, these 4 patient groups represent different levels of symptom risk whose interpretation varies across gender, for classes 3 and 4. Two character TCIs self-directedness (SD) and self-transcendence (ST) were significant in predicting class status. In general higher ST was associated with higher distress, and higher SD with lower distress, but their impact varied across gender in class 4 and across gender to a less extent in the low risk class 2. Earlier a LCA by Leigh et al., (2012) found 3 latent classes for which reward dependence (RD) and cooperativeness $(C)$ were significant predictors for pre and post treatment SCLs, respectively. The impact of RD and C differed across the sexes. Similarly a LCA for the pre and post data separately, but with gender as a grouped predictor (not reported by Leigh et al., 2012) found 3 latent classes, but for this model SD and ST were significant predictors of latent class status. Our LTA analysis here, which allows pre and post treatment transitions between classes, and gender as a grouping variable, also found SD and ST were significant predictors of class, but four classes was found as the optimal number of groups based on goodness of fit statistics. The LTA results reported also agree, in part, with preliminary univariate Generalised 
Additive Model for Location, Scale and Shape (GAMLSS) models for each SCL based on combined pre and post treatment data (Hudson et al., 2015), which allow for non-linearity between TCIs and symptoms, and for interactions between TCI with gender and time. These build on earlier GAMS models by Turner (2004) which found significant differences in TCI predictors of distress across gender, but for the pre and post data separately. GAMLSS (Hudson et al., 2015) found significant interactions between gender and 5 TCIs, SD, ST, C, RD and P. Gender-specific lower and upper TCI cut-points for the non-linear TCIs were identified, which define regions of the given TCI, above and below which, the directionality of the association between TCI and SCL changed. These support LTA findings that SD and ST impact symptoms over time differently according to sex and class. Specifically Hudson et al., (2015) showed significant ST*gender interactions for depression and OC, which increased with ST between $0.2-0.7$ more for males. However at higher scores of ST $>0.7$ depression and OC in the males actually improved with increasing ST. Also a significant SD*gender interaction for four SCLs, IS, $\mathrm{OC}, \mathrm{AH}$ and psychoticism $(\mathrm{P})$ was found, with these SCLs improving more for females with increasing SD; for lower SD $<0.3$ all but IS, did not improve with increasing SD for males. A significant RD*gender interaction with depression, anxiety, $\mathrm{P}$ and OC showed these SCLs decreased with increasing RD $>0.5$ with similar rates across gender, but for RD scores above the median RD $>0.8$ these SCLs actually increased with RD for females. There was a significant $\mathrm{C}^{*}$ gender interaction for somatisation (S), $\mathrm{P}$ and $\mathrm{AH}$, which decreased with increasing $\mathrm{C}$ for $\mathrm{C}>0.55$ - more for males. But for $\mathrm{C}$ in the lower range $\mathrm{C}<0.50, \mathrm{~S}$ and $\mathrm{P}$ reversed directionality increasing with higher $\mathrm{C}$ more for males. AH decreased with increasing $\mathrm{C}>0.4$ across the sexes.

Given the TCI cut-points future work will examine other covariates such as age, socio-economic class. Another direction will be a study of polychotomous SCL outcomes and TCIs to identify differing class structure, and test for changing relationships between TCIs and SCLs across gender, time and patient clusters of Lee et al. (2012) which have different SCL profiles post treatment. GAMLSS models provide a specific sets of TCI predictors to better assess distress risk, symptom by symptom across treatment and gender, as do our LTA models which are a multivariate SCL approach. Both studies show the value of gender-specific models for psychological distress.

\section{REFERENCES}

Cloninger, C. (2008). The psychobiological theory of temperament and character: comment on Farmer and Goldberg. Psychological Assessment, 20, 292-304

Collins, L.M., and S.T. Lanza (2010). Latent class and latent transition analysis with applications in the social, behavioural and health sciences, Wiley Series in Probability and Statistics, ed. D.J. Balding, et al., Hoboken. John Wiley \& Sons, New Jersey.

Derogatis, L.R., (1983). SCL-90-R, Administration, scoring and procedures, Manual II for the Revised Version of the SCL-90. John Hopkins University Press, Baltimore.

Joyce, P.R., R.T. Mulder, S.E. Luty, P.F. Sullivan, J.M. McKenzie, R.M. Abbott, and I.F. Stevens (2002).

Patterns and predictors of remission, response and recovery in major depression treated with fluoxetine or nortriptyline. Australian and New Zealand Journal of Psychiatry, 36,384-391.

Hudson, I.L., R.M. Turner, and P.R. Joyce (2015). Gender specific prediction of psychological distress symptoms using Cloninger's personality traits: a GAMLSS approach (in prep).

Lee, S., I.L. Hudson, S. Lee, R. Turner, and P. R. Joyce (2012). Visualizing improved prognosis in psychiatric treatment via mixtures. Australian Statistical Conference. SSAI, pp. 131. Adelaide, Australia, Jul 9-12.

Leigh, L., I.L. Hudson, R. Turner, and P. Joyce (2012). Classifying multivariate risk profiles of depression symptoms using Cloninger's temperament and character traits: A latent class model approach. Australian Statistical Conference. Statistical Society of Australia, pp. 132, Adelaide, Australia, Jul 9-12.

Turner, R.M., I.L. Hudson, P.H. Butler, and P.R. Joyce (2003). Brain function and personality in normal males: a SPECT study using statistical parametric mapping. NeuroImage, 19, 1145-1162.

Joyce, P.R., and P. Mitchell (eds) (2004). Mood disorders: recognition and treatment. UNSW Press, Australia.

Turner, R.M. (2004). Independent component analysis of personality and symptoms of depression and statistical parametric mapping of personality and brain function. $\mathrm{PhD}$ thesis in Mathematical Statistics, University of Canterbury, Christchurch, New Zealand. 